\title{
Breast feeding and vitamin A deficiency among children attending a diarrhoea treatment centre in Bangladesh: a case-control study ) (
}

\author{
Dilip/Mahalanabis
}

\begin{abstract}
Objective-To determine the effect of breast feeding on the risk of xerophthalmia in children aged 6 months to 3 years attending a diarrhoea treatment centre in Bangladesh.

Design-Case-control study based on stratified analysis (Mantel-Haenszel) and multivariate analysis (logistic regression) of data from a treatment centre based surveillance system.

Setting-A large diarrhoea treatment centre in Dhaka, Bangladesh.
\end{abstract}

Patients -2687 children aged 6 months to 3 years representing a $4 \%$ systematic sample of all children in this age group treated yearly at the centre over three consecutive years. 66 of the children were cases of xerophthalmia (that is, they had Bitot's spots or corneal lesions or night blindness or night blindness plus conjunctival xerosis or any combination of these) and the remaining 2621 did not have signs or symptoms of vitamin $\mathbf{A}$ deficiency. This second group served as controls.

Main outcome measure-Xerophthalmia and breast fed at onset of diarrhoea or presentation.

Results - The odds ratio relating breast feeding to vitamin A deficiency after adjustment for a large number of confounding variables $(0 \cdot 26)(95 \%$ confidence interval 0.14 to 0.49 ); $p<0.001$ ) reflected a $74 \%$ reduction in the risk of vitamin $A$ deficiency among breast fed children. The estimated reduction of risk did not decline with age, and some $49 \%$ of children aged 24-35 months were still being breast fed. The odds ratio relating breast feeding to xerophthalmia in the third year of life $(0.35(95 \%$ confidence interval 0.35 to 0.86 ) reflected a $65 \%$ reduced risk of vitamin A deficiency. Other important risk factors or prognostic indicators for xerophthalmia as identified by multivariate analysis were recent measles, prolonged diarrhoea, severe protein energy malnutrition, and poor socioeconomic state.

Conclusions-These results indicate that breast feeding was associated with a substantial reduction of the risk of vitamin A deficiency extending to the third year of life and support the recommendation that mothers in developing countries should be advised to breast feed for as long as possible.

\section{Introduction}

Nutritional blindness due to vitamin A deficiency is an important public health problem in Bangladesh ${ }^{12}$ as in many other developing countries. In a prospective study in Bangladesh the prevalence of active corneal lesions was 10 times the 1/10000 World Health Organisation's public health threshold.' Every year worldwide an estimated 10 million children under the age of 6 years develop some degree of xerophthalmia and about a million of them go blind. ${ }^{3}$ Furthermore, two studies in Indonesia showed that children with mild signs of vitamin A deficiency have a substantially higher mortality than children who do not have such signs $^{4}$ and that vitamin $\mathrm{A}$ supplementation at the community level reduces mortality by $30 \%$ compared with controls. ${ }^{5}$ Hence evaluation of factors which protect a child from vitamin A deficiency assumes great importance. I report a case-control study in which multivariate analysis was used to examine the effect of breast feeding on the risk of xerophthalmia in children attending a diarrhoea treatment centre in Bangladesh.

\section{Subjects and methods}

Detailed information on a systematic sample of $4 \%$ of all patients - that is, every 25th of 60000 to 90000 patients a year - attending a diarrhoea treatment centre of the International Centre for Diarrhoeal Disease Research, Bangladesh (ICDDR,B), in Dhaka has been maintained since 1980 as a special research project on a hospital based surveillance system. ${ }^{6}$ Methods of data collection have been described. ${ }^{6}$ Patients are treated in an outpatient area. Those who require further care are admitted to a short stay treatment unit or to a hospital ward if longer stay or more complicated care is thought necessary.

After initial care the patient or a guardian is interviewed by a specially trained health assistant and uniform information on sociodemographic features, antecedent history, prevailing symptoms, and previous treatment recorded. A doctor performs a physical examination at the time of admission and records the data on a form. Each patient is weighed and measured before discharge. A rectal swab for culture and a stool sample for microscopy are sent to the laboratory. If a patient is admitted additional details are recorded on a form until discharge. Data forms are then carefully checked by experienced health workers and entered into a mainframe computer (IBM System 34 ). Specially trained staff have been assigned exclusively to this project.

A large poor community in the city of metropolitan Dhaka uses this centre for the primary treatment of diarrhoea as services are free (including meals) and no other hospital in the city admits patients with diarrhoea or dysentery. Therefore, these patients largely represent the urban poor. All children aged 6-35 months entered into the surveillance system during 1983-5 were included in this analysis. A total of 3208 children were evaluated. Patients with Bitot's spots or corneal lesions or night blindness plus conjunctival xerosis or night blindness and any combination of these were cases of vitamin A deficiency. In an earlier study of this population night blindness was a useful indicator of vitamin A deficiency.? To minimise misclassification bias 161 patients with only conjunctival xerosis were excluded from the analysis. Controls were all other children in whom signs of xerophthalmia were absent.

\section{STATISTICS AND CONFOUNDING VARIABLES}

Data from the mainframe computer were downloaded into a microcomputer and validated by visual and logical checks by using the statistical package for the social sciences (SPSS) PC + and then analysed for crude odds ratio and Mantel-Haenszel statistics ${ }^{x}$ by using the software EPI INFO (Centers for Disease Control, Atlanta, Georgia). Unconditional logistic 
regression analyses were carried out with the software EGRET (epidemiological graphics, estimation, and testing package).

For calculating sample size we considered the effect of lack of breast feeding on the risk of xerophthalmia and wished to detect an odds ratio of 3.0 with a power of $80 \%$ at the $5 \%$ level of significance. One fifth of the mothers did not breast feed, so we needed 87 cases (including an allowance of $25 \%$ for confounders) for one control per case. For multivariate analysis 66 cases and 2621 controls were available. We were therefore restricted to 66 cases but had many more controls. If we included all the controls we then needed only 44 cases. If we wished to detect a smaller odds ratio of 2.5 then the calculated number of cases was 65 when using all available controls. Therefore, with the available number of cases and controls we calculated that we should be able to detect an odds ratio of 2.5 for the effect of lack of breast feeding on xerophthalmia in these children. In other words, using all the available cases and controls we should be able to detect a reduction in the risk of vitamin A deficiency of $60 \%$ or more.

A crude odds ratio was calculated for the association between breast feeding and vitamin A deficiency signs (xerophthalmia). This $2 \times 2$ table was then stratified by each of the likely confounding variables one at a time and a Mantel-Haenszel summary odds ratio calculated and compared with the crude odds ratio. ${ }^{8}$ Confounders for which the Mantel-Haenszel odds ratio deviated from the crude odds ratio were then entered into an unconditional logistic regression model. In addition, variables thought to be biologically plausible confounders but which did not meet the above criteria were also entered into the model. The logistic regression analysis included 2687 children (66 cases, 2621 controls). Of 360 patients who could not be included because of incomplete records, 11 had xerophthalmia and in 70 information on breast feeding was missing. Breast fed children were those who were either exclusively breast fed or receiving other foods in addition. Non-breast fed children were those who were not receiving any breast milk at the time of onset of the current illness.

Confounding variables adjusted for (see table) were age, nutritional state, family income, duration of diarrhoea, mother's education, use of contaminated surface water for any purpose, recent history of measles, concomitant chest infection, dehydration,

Multivariate analysis of breast feeding and reduced risk of vitamin A deficiency in children aged 6-35 month attending treatment centre for diarrhoea in Dhaka, Banglades $h^{\star}$

\begin{tabular}{|c|c|c|}
\hline Explanatory variable ${ }^{\star}$ & $\begin{array}{l}\text { Odds ratio } \\
(95 \% \text { confidence interval })\end{array}$ & $\mathrm{p}$ Value \\
\hline Breast fed $\nmid$ (variable of interest) & $0.26(0.14$ to $0 \cdot 49)$ & $<0.001$ \\
\hline \multicolumn{3}{|l|}{ Older age: } \\
\hline 24-35 months & $3.8(1.6$ to 8.9$)$ & 0.002 \\
\hline $\begin{array}{l}12-23 \text { months } \\
\text { (Reference category } 6-11 \text { months) }\end{array}$ & $1.8(0.8$ to 4.0$)$ & $0 \cdot 163$ \\
\hline \multirow{2}{*}{\multicolumn{3}{|c|}{ Malnutrition ( $\%$ National Centre for Health Statistics median): }} \\
\hline & & \\
\hline $\begin{array}{l}60-70 \% \text { weight for age } \\
(\text { Reference category }>70 \%)\end{array}$ & $0.72(0.28$ to 1.8$)$ & 0.5 \\
\hline Poverty (family income $<£ 17$ per month) & $2 \cdot 64(1.3$ to $5 \cdot 5)$ & 0.009 \\
\hline \multicolumn{3}{|l|}{ Diarrhoea duration: } \\
\hline$>14$ days & $3.5(1.8$ to $6 \cdot 8)$ & $<0.001$ \\
\hline 10-14 days & $5 \cdot 3(2 \cdot 2$ to $12 \cdot 5)$ & $<0.001$ \\
\hline \multicolumn{3}{|l|}{ (Reference category $\leqslant 9$ days $)$} \\
\hline Used contaminated surface water for any purpose & $1.6(0.9$ to 3.0$)$ & $0 \cdot 123$ \\
\hline Lack of mother's education (no formal school education) & $2 \cdot 2(0 \cdot 9$ to $5 \cdot 1)$ & 0.076 \\
\hline Measles in recent past & $3.5(1.6$ to 7.6$)$ & 0.002 \\
\hline Concomitant chest infection & $2 \cdot 7(1 \cdot 3$ to $6 \cdot 1)$ & 0.006 \\
\hline Presence of stomatitis & $1.7(0.7$ to $4 \cdot 2)$ & $0 \cdot 222$ \\
\hline Moderate or severe dehydration & $0.68(0.35$ to 1.26$)$ & $0 \cdot 218$ \\
\hline Female sex & $0.79(0.43$ to 1.46$)$ & 0.461 \\
\hline Shigellosis & $1 \cdot 1(0 \cdot 49$ to $2 \cdot 26)$ & 0.889 \\
\hline
\end{tabular}

Constant term $=-5.906$

Likelihood ratio statistic on 16 degrees of freedom $=3331.62 ; p<0.001$

^Logistic regression model. Xerophthalmia was dependent variable.

tDefined as children who were exclusively breast fed or who continued breast feeding but were receiving other foods at time of attendance at treatment centre or up to time of onset of current illness.

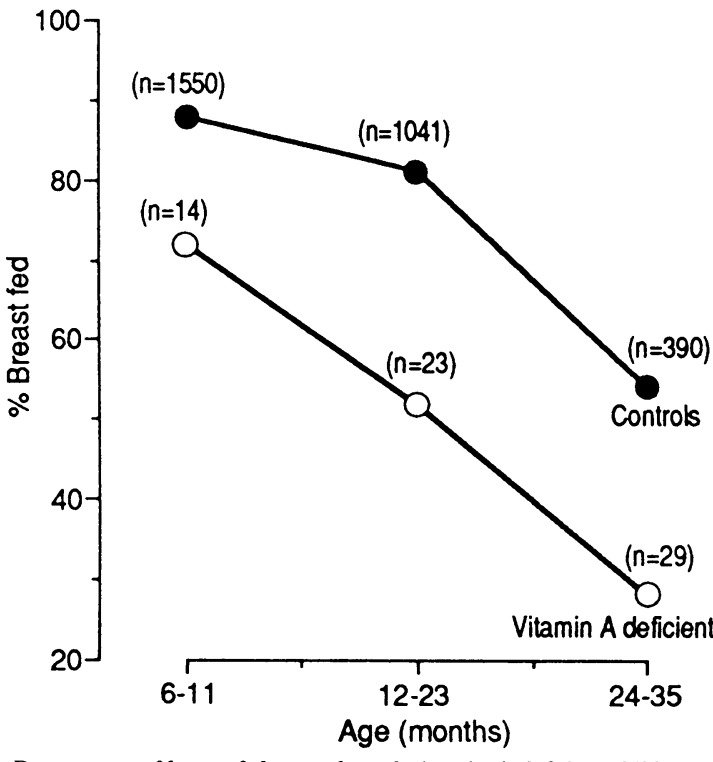

Percentages of breast fed controls and vitamin A deficient children in three age groups. Crude odds ratios for association between breas feeding and vitamin A deficiency in age groups 6-11 months, 12-23 months, and $24-35$ months were $0 \cdot 33,0 \cdot 27$, and 0.35 respectively. Age stratified Mantel-Haenszel summary odds ratio for association between breast feeding and vitamin $A$ deficiency $0 \cdot 32$ (95\% confidence interval $0 \cdot 18$ to $0 \cdot 55$

presence of stomatitis, sex, and positive stoool culture for shigellas.

\section{Results}

Of the 66 children $(2 \cdot 1 \%$ of all 3208 evaluated) with xerophthalmia, eight had corneal lesions, 12 Bitot's spots, three both Bitot's spots and night blindness, 23 conjunctival xerosis plus night blindness, and 20 night blindness alone. The youngest child with night blindness was aged 12 months. Overall, roughly $2415(81 \%)$ controls and $30(46 \%)$ vitamin A deficient children were currently being breast fed. The unadjusted odds ratio indicated that breast feeding was associated with a reduced risk of vitamin A deficiency signs of about $80 \%$ (odds ratio $0 \cdot 20 ; 95 \%$ confidence interval $0 \cdot 12$ to $0 \cdot 34$ ) After adjusting for a large number of confounders in the logistic regression model (table) breast feeding was still associated with a $74 \%$ reduction (odds ratio $0 \cdot 26$ $95 \%$ confidence interval $0 \cdot 14$ to $0 \cdot 49$ ) in vitamin A deficiency signs.

In children with increasing age the proportion with vitamin A deficiency signs increased and the rate of breast feeding decreased. Age stratified breast feeding rates, however, were consistently higher in controls than in vitamin A deficient children (figure). The odds ratios relating breast feeding to vitamin A deficiency for age groups $6-11$ months $(0.33 ; 95 \%$ confidence interval 0.09 to 1.27$), 12-23$ months $(0.27 ; 0.11$ to $0.67)$, and $24-35$ months $(0.35 ; 0.35$ to 0.86$)$ reflected a $67-73 \%$ reduced risk of vitamin A deficiency in breast fed children in these age categories.

In children who did not have a recent history of measles the odds ratio relating breast feeding to xerophthalmia was $0 \cdot 17$ (95\% confidence interval $0 \cdot 10$ to $0 \cdot 31$ ), which reflected an $83 \%$ reduced risk. In those who had had measles recently, however, the odds ratio was $0.42(95 \%$ confidence interval 0.10 to 1.81$)$, reflecting only a $58 \%$ reduced risk and indicating that recent measles could overwhelm the protective effect of breast feeding. Similarly, in children with a short duration of diarrhoea (nine days or less) the odds ratio was $0.18(95 \%$ confidence interval 0.09 to 0.04$)$ whereas in persistent diarrhoea (duration over 14 days) the odds ratio was $0 \cdot 30(0 \cdot 12$ to $0 \cdot 76)$.

The same trend was shown for concomitant chest infection. In children without chest infection the odds 
ratio was 0.17 ( $95 \%$ confidence interval 0.09 to 0.31 ) whereas in those with chest infection the odds ratio was $0.30(0.09$ to 1.01$)$. In children with severe protein energy malnutrition (less than $60 \%$ of weight for age) the odds ratio was $0 \cdot 27$ ( $95 \%$ confidence interval $0 \cdot 14$ to 0.54 ) whereas in better nourished children (over $70 \%$ of weight for age $)$ the odds ratio was $0 \cdot 10(0.03$ to $0 \cdot 37$ ). As expected, in the multivariate analysis these variables were also significant risk factors for xerophthalmia.

\section{Discussion}

Breast feeding is generally regarded as protective against xerophthalmia in infants and young children." At least two reports have provided evidence on the association between lack of breast feeding and the risk of xerophthalmia in children. In Indonesia 358 children under 5 years of age with Bitot's spots were compared with 340 age and sex matched controls and also with 34576 clinically normal children under 5 years of age representing $96 \%$ of urban slum and rural preschool children. The authors found that breast feeding was less common in the children with Bitot's spots than in controls $(\mathrm{p}<0 \cdot 001)$."

We further analysed these data of Tarwotjo $e t a l^{10}$ using a Mantel-Haenszel procedure ${ }^{8}$ and the results were revealing. The age stratified summary odds ratio (Mantel-Haenszel) for an association between Bitot's spots and lack of breast feeding was $2.82(95 \%$ confidence interval 1.52 to 5.74 ) when cases were compared with age and sex matched controls and 3.8 (2.96 to $8 \cdot 8)$ when compared with the much larger group of community controls. These findings indicated a threefold to fourfold increased risk of vitamin A deficiency in non-breast fed children. Confounding effects of variables other than age and sex were not evaluated. The results, however, were similar to ours from logistic regression analysis of the surveillance data-namely, lack of breast feeding was associated with about a fourfold increased risk of vitamin A deficiency after adjusting for a large number of confounders.

In a case-control study on the association of xerophthalmia with breast feeding and weaning patterns in children aged 24-71 months in southern Malawi 152 children with xerophthalmia were compared with 151 clinically normal children matched for age (within 12 months), sex, and village of residence. ${ }^{11}$ Children with xerophthalmia had begun weaning with porridge sooner $(\mathrm{p}=0.05)$, had stopped breast feeding earlier ( $\mathrm{p}<0.01$ for $24-47$ months old $),$ had a shorter weaning interval - that is, the duration between the introduction of porridge and stopping breast feeding $-(\mathrm{p}<0.005)$, and had been fully weaned longer $(p<0.025)$ than controls. These findings imply a protective role of breast feeding against xerophthalmia in early childhood but give no estimate of its magnitude.

\section{EFFECT OF POSSIBLE MISCLASSIFICATION OF CASES}

This study included children with night blindness only among the cases of vitamin A deficiency, which raises the possibility of misclassification of cases. Three questions must therefore be answered, concerning $(a)$ the reliability of ascertaining night blindness in young children, $(b)$ the reliability of night blindness as an indicator of vitamin A deficiency, and $(c)$ the effect of misclassification on the analysis of results. The youngest child in the series with night blindness was 1 year old.

Night blindness (commonly called "Rat kana" locally) is well recognised in the Indian subcontinent. On careful questioning of parents (as routinely done by experienced interviewers in this surveillance project) a history of night blindness in young children is usually qualified by statements that the child is unable to find or reach for familiar objects at night. Hence the population's awareness of the condition together with careful interview techniques assure a high degree of accuracy in ascertaining night blindness. Similar findings were reported by Sinha and Bang in a prospective community study. ${ }^{12}$

Night blindness is a recognised complication of xerophthalmia (coded XN in the international classification), ${ }^{12 a}$ and one validation study has been conducted in this population.' In the absence of practical and objective measures of dark adaptation (a function known to be affected by vitamin A deficiency) and given that vitamin A deficiency is hyperendemic in this population a carefully elicited history of night blindness in the absence of any eye disease may be considered to be a generally reliable indicator of vitamin A deficiency. It remains possible, however, that a few children with night blindness may not have had vitamin A deficiency and were therefore misclassified as cases.

What would be the effect of misclassification on the results of this case-control analysis? In general terms random and independent errors of classification tend to diminish the apparent degree of association between two variables. ${ }^{1314}$ The effect of classifying a non-case as a case would be to bias the estimate of the odds ratio towards unity. ${ }^{15}$ This will always be true if the misclassification is non-differential-that is, the probability of a child being wrongly classified as a case being independent of whether the child is breast fed or not. Hence if such misclassification occurred in this study the measured protection by breast feeding would be underestimated.

This paper reports a semiquantitative estimate of the role of breast feeding in preventing xerophthalmia in children using multivariate analysis and adjusting for a large number of confounding variables. Subjects were an unbiased sample of patients attending a treatment centre for diarrhoea which provides primary care to a large poor urban population. In the absence of any other free hospital in the city which admits patients with diarrhoea and dysentery an overwhelming majority of the urban poor with diarrhoea have no option but to attend this treatment centre. The results of the analysis are therefore generally applicable to the urban poor.

Vitamin A deficiency is hyperendemic in Bangladesh.' Hence the estimated association between breast feeding and vitamin A deficiency was likely to be conservative because some children in the control group probably had subclinical vitamin A deficiency. Furthermore, the control group included children who had stopped breast feeding at variable intervals after birth, so that some of those who had stopped recently may have been partially protected. ${ }^{.1}$

\section{CAUSE AND EFFECT RELATION}

In Bangladesh a large proportion of children are breast fed for long periods. In this sample nearly $49 \%$ of children aged 24-35 months were still being breast fed. Thus in terms of the whole population the effect of breast feeding in reducing vitamin A deficiency should be substantial provided that a cause and effect relation exists. A cause and effect relation is plausible because $(a)$ breast feeding would precede the development of vitamin A deficiency for the obvious reason that breast feeding starts soon after birth, $(b)$ mothers' milk contains a substantial amount of vitamin $A,{ }^{16}$ and (c) even older breast fed children may be receiving as much as $400-600 \mathrm{ml}$ mothers' milk a day. ${ }^{17-21}$

A recent study showed that the vitamin A concentration in the breast milk of poor mothers in periurban Bangladesh may be as high as $1 \cdot 0-1 \cdot 4 \mu \mathrm{mol} / 1,{ }^{22}$ so an estimated average intake of, say, $400 \mathrm{ml}$ breast milk a day could provide at least $30-40 \%$ of a child's daily 
requirement of vitamin A. In Bangladesh green leafy vegetables are considered inappropriate food for young children, but not for adults. Also, these food items are regarded as inferior. In a male dominated society, therefore, women are likely to consume more of green leafy vegetables while men consume more of socially attractive foods.

This case-control study used an analytical approach to evaluate the role of breast feeding. The multivariate analysis (table), however, also showed several other factors to have a strong association with xerophthalmia in children. A confounding variable was also a risk factor for the study disease (xerophthalmia) and was associated with the study variable of primary interest (breast feeding). The odds ratio associated with any particular confounding variable in the logistic mode (table) was therefore interpreted to indicate the degree of its association with xerophthalmia. The risk of xerophthalmia was 3.5 times higher $(p=0.002)$ in children with a recent history of measles, 3.8 times higher in severely malnourished children $(p<0 \cdot 001)$, and 2.7 times higher $(p=0.006)$ in children with concomitant chest infection. With respect to socioeconomic factors xerophthalmia was 2.6 times higher $(p=0.009)$ in children from low income families and $2 \cdot 2$ times higher $(p=0.076)$ in children of illiterate mothers. The estimated relative risk for each of the above factors was adjusted for all other factors in the logistic model. This analysis therefore identified several other important risk factors or prognostic indicators for xerophthalmia in children. Altogether $30(46 \%)$ of the children with xerophthalmia were being breast fed. Possible explanations for lack of protection in these children included infections like measles and acute respiratory infections, which might overwhelm the protective effect; that they were receiving much smaller amounts of breast milk; and that the vitamin A concentration in the mothers' milk was low (presumably owing to deficient intake by the mother).

\section{Conclusion}

In conclusion a strong positive association was found between breast feeding and lack of xerophthalmia up to 3 years of age after adjusting for a large number of likely confounders. A cause and effect relation was suggested by the temporal sequence, consistency with other reports, strength of the association after adjusting for likely confounders, and obvious biological plausibility (the vitamin A concentration in mothers' milk). Breast feeding may also indirectly prevent vitamin A deficiency by protecting the child against recurrent infections like diarrhoea and acute respiratory tract infection.

In this poor urban population of Bangladesh the prevalence of breast feeding up to the third year of life was remarkably high. It is therefore likely that any substantial reduction in the rate of prolonged breast feeding might lead to a pronounced increase in the prevalence of xerophthalmia. This has important implications for the promotion of breast feeding and its duration and supports the conclusion of a recent review on breast feeding and child survival that underprivileged mothers should be advised to breast feed for as long as possible. ${ }^{23}$ To enable mothers to effectively breast feed for a long period it is important to promote child spacing, which has other obvious benefits. A recent suggestion that breast feeding should stop at around 18 months $^{24}$ is not appropriate for Bangladesh and other developing countries where vitamin A deficiency is endemic.
This study was supported by the Swiss Development Cooperation and the ICIDDR,B. The ICDDR, B is supported by countries and agencies which share its concern for health problems in developing countries. Current donors include the governmental aid agencies of Australia, Bangladesh, Belgium, Canada, Denmark, France, Japan, The Netherlands, Norway, Sweden, Switzerland, the United Kingdom, and the United States; international organisations including the United Nations Capital Development Fund, the United Nations Development Programme, the United Nations Children's Fund, and the World Health Organisation; and private foundations including the Ford Foundation and the Sasakawa Foundation.

I thank Dr Abbas Bhuiya, Dr D Habte (ICDDR,B), and Dr Kenneth Brown (University of California, Davis) for critically reviewing the manuscript and offering helpful suggestions, Mr Abul Hasnat for help in data management and analysis, and Mr Nurur Rahman for data collection and validation. I also thank Ms Loretta Saldanha-Ansari for typing and editing the manuscript.

1 Institute of Public Health Nurrition and Helen Keller International. Bangladesh national nutritional blindness study. Key results. Dhaka, New York: IPHN, HKI, 1985.

2 Cohen N, Jalil MA, Rahman H, Matin MA, Sprague J, Islam J, et al Landholding, wealth and risk of hlinding malnutrition in rural Bangladeshi households. Soc Sici Med 1985;21:1269-72.

3 Habte D. Global dimensions of vitamin A deficiency. In: Morck TA, Lindsay L, eds. Proceedings of national symposium and XIII international vitumin . consultatize group meeting, Kathmandu, Nepal. Washington, DC: Nutrition Foundation, 1989:7-8

+ Sommer A, Tarwotjo I, Hussaini G, Susanto D. Increased mortality in children with mild vitamin $A$ deficiency. Lancet 1983;ii:585-8.

5 Sommer A, Tarwotjo I, Djunaedi E, West KP Jr, Loeden AA, Tilden R, et al. Impact of vitamin A supplementation on childhood mortality: a randomised controlled community trial. Lancet 1986;:11169-73.

6 Stoll BJ, Glass RI, Huq MI, Khan MU, Holt JE, Banu H. Surveillance of patients attending a diarrhoeal disease hospital in Bangladesh. $B M J$ 1982:285:1185-8

7 Stoll BJ, Banu H, Kabir I, Molla A. Nightblindness and vitamin A deficiency in children attending a diarrhoeal disease hospital in Bangladesh. I Trop Pediatr 1985;31:36-9.

8 Mantel N, Haenszel W. Statistical aspects of the analysis of data from retrospective studies of disease. F Natl Cancer Inst 1959;22:719-48.

9 World Health Organisation. Vitamin A deficiency and xerophthalmia. Report of a joint WHO/USAID meeting. WHO Tech Rep Ser 1976; No590:35.

10 Tarwotjo I, Sommer A, Soegiharto T, Susanto D, Muhilal. Dietary practices and xerophthalmia among Indonesian children. Am f (ilin Nutr practices and $\mathrm{x}$

11 West KP Jr, Chirambo M, Katz J, Sommer A. Breast-feeding, weaning patterns, and the risk of xerophthalmia in southern Malawi. Am $f$ Clin Nur $1986 ; 4+690-7$

12 Sinha DP, Bang $\mathrm{FB}$. Seasonal variation in signs of vitamin A deficiency in rural West Bengal children. Lancet 1973;ii:228-31.

12a Sommer A. McLaren DS, Olson JA. Assessment of vitamin A status, IVACG Task force on Assessment. In: (iuidelines for the eradication of vilamin A deficiency and xerophthalmia. A report of the International Vitamin A Consultative (iroup (IVACG). Washington, DC: The Nutrition Foundation, 1977:1-7.

13 Bross IDJ. Misclassification in $2 \times 2$ tables. Biometrics 1954;10:478-86.

14 Newell DJ. Misclassification in $2 \times 2$ tables. Biometrics 1963:19:187-8.

15 Schlesselman JJ. Case-control studies: design, conduct, analysis. Oxford: Oxford University Press, 1982:137-40.

16 Gebre-Medhin M, Vahlquist A, Hofvander Y, Uppsall L, Vahlquist B. Breast milk composition in Ethiopian and Swedish mothers. I. Vitamin A and $\beta$ carotene. Am f Clin Nutr 1976;29:441-51.

17 Van Steenbergen WM, Kusin JA, Vans Rens MM. Lactation performance of Akamba mothers, Kenya. Breast-feeding behaviour, breast milk yield and composition. F Trop Pediatr 1981;27:155-61.

18 Roy SK, Rabbani GH, Black RE. Oral rehydration solution safely used in breast-fed children without additional water. F Trop.Med Hyg 1984;87:11-3.

19 Vis H-L. Hennart P, Ruchababisha M. Some issues in breast-feeding in deprived rural areas: maternal nutrition and breast-feeding in the Kivu, Zaire. Assignment Children 1981;(55/6): 183-200.

20 Becroft TC. Child-rearing practices in the highlands of New Guinea: general features. Med F A Ast 1967;ii:810-3.

21 Brown KH, Black RE, Becker S, Nahar S, Sawyer J. Consumption of foods and nutritients by weanlings in rural Bangladesh. Am f Clin Nutr 1982;36 (5):878-89.

22 Roy SK, Islam A, Molla A, Akramuzzaman SM. Dynamics of vitamin A levels in the breastmilk of mothers of low socioeconomic status in Bangladesh. In: Darnton Hill I, ed. Vitamin A deficiency in Bangladesh: prevention and control. Dhaka: Helen Keller International, Bangladesh and Voluntary Health Services Society, 1989:107-15.

23 Dualeh KA, Henry FJ. Breastmilk - the life saver: observations from recent studies. Food and Nutrition Bulletin 1989;11:43-6.

24 Brakohiapa LA, Yartey J, Bille A, Harrison E, Qunsah E, Armar MA, ct al. Does prolonged breast-feeding adversely affect a child's nutritional status? Lancet 1988;ii:416-8.

Accepted 13 Jun' 1991 